$\begin{array}{ll}\text { Research Square } & \text { Preprints are preliminary reports that have not undergone peer review. } \\ \text { They should not be considered conclusive, used to inform clinical practice, } \\ \text { or referenced by the media as validated information. }\end{array}$

\title{
Association Between Serum Oestradiol Level on the Human Chorionic Gonadotrophin Administration Day and Neonatal Birthweight After in Vitro Fertilization-embryo Transfer: A Retrospective Study of 3659 Singleton Live Births
}

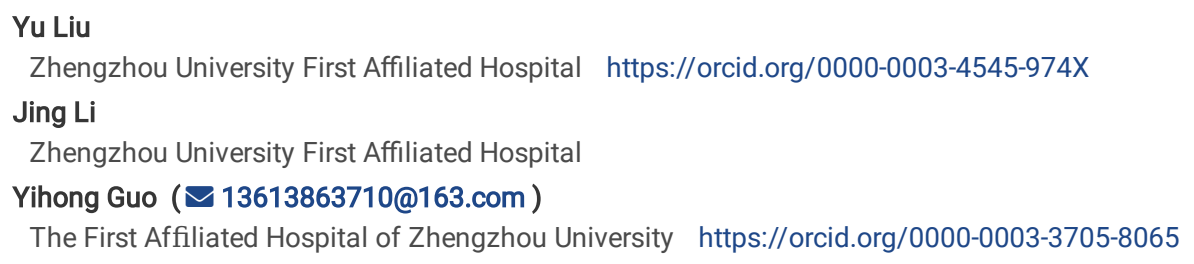




\section{Abstract}

Background

Oestradiol, an important hormone in follicular development and endometrial receptivity, is closely related to clinical outcomes of fresh in vitro fertilizationembryo transfer (IVF-ET) cycles. A supraphysiologic E2 level is inevitable during controlled ovarian hyper-stimulation ( $\mathrm{COH}$ ), and its effect on the outcome of IVF-ET is controversial. The aim of this retrospective study is to evaluate the association between elevated serum oestradiol (E2) levels on the day of human chorionic gonadotrophin (hCG) administration and neonatal birthweight after IVF-ET cycles.

Methods

The data of 3659 infertile patients with fresh IVF-ET cycles were analysed retrospectively between August 2009 and February 2017 in First Hospital of Zhengzhou University. Patients were categorized by serum E2 levels on the day of hCG administration into six groups: group 1 (serum $\mathrm{E}_{2}$ levels $\leq 1000 \mathrm{pg} / \mathrm{mL}$, $\mathrm{n}=230$ ), group 2 (serum E2 levels between 1001 and $2000 \mathrm{pg} / \mathrm{mL}, \mathrm{n}=524$ ), group 3 (serum $E_{2}$ levels between 2001 and $3000 \mathrm{pg} / \mathrm{mL}, \mathrm{n}=783$ ), group 4 (serum $E_{2}$ levels between 3001 and $4000 \mathrm{pg} / \mathrm{mL}, \mathrm{n}=721$ ), group 5 (serum E2 levels between 4001 and $5000 \mathrm{pg} / \mathrm{mL}, \mathrm{n}=548$ ), and group 6 (serum E2 levels > 5000 $\mathrm{pg} / \mathrm{mL}, \mathrm{n}=852$ ). Univariate linear regression was used to evaluate the independent correlation between each factor and outcome index. Multiple logistic regression was used to adjust for confounding factors.

Results

The LBW rates were as follows: 3.0\% (group 1), 2.9\% (group 2), 1.9\% (group 3), 2.9\% (group 4), and 2.0\% (group 6) $(P=0.629)$, respectively. There were no statistically significant differences in the incidences of neonatal LBW among the six groups. We did not detect an association between peak serum E2 level during ovarian stimulation and neonatal birthweight after IVF-ET.

Conclusion

The results of this retrospective cohort study showed that serum E2 peak levels during ovarian stimulation were not associated with birth weight during IVF cycles. In addition, no association was found between higher E2 levels and increased LBW risk. Our observations suggest that the hyper-oestrogenic milieu during COS does not seem to have adverse effects on the birthweight of offspring after IVF.

\section{Background}

Since the birth of Louise Brown in the UK in 1978 [1], the use of in vitro fertilization (IVF) to treat infertility has steadily increased, with more than seven million children born worldwide [2]. In vitro fertilization-embryo transfer (IVF-ET) is the main component of assisted reproductive technology (ART) and the most effective method to help infertile patients conceive. However, compared with infants conceived naturally, IVF-conceived infants, whether in a multiple or singleton pregnancy, usually have a higher risk of adverse perinatal outcomes, including low birth weight (LBW) and small for gestational age (SGA) [3]. It is still unclear whether these increased risks are due to the inherent characteristics of infertility, the IVF treatment itself, or a combination of both [4]. Controlled ovarian hyper-stimulation $(\mathrm{COH})$ is considered a key determinant of IVF success, wherein multiple dominant follicles are recruited to increase the number of eggs for harvest in a single cycle. During the $\mathrm{COH}$ cycle, serum oestradiol (E2) levels can be increased more than tenfold that of a natural cycle. However, recent studies suggest that reproductive physiological E2 levels during $\mathrm{COH}$ may produce an unsatisfactory peri-implantation uterine environment, which leads to placental abnormalities and, ultimately, to adverse neonatal outcomes, such as preeclampsia, LBW, and SGA [3]. However, the effects of exposure to such high E2 levels on the day of human chorionic gonadotropin (hCG) injection during the IVF cycle are still unclear [5]. Recent evidence suggests that serum E2 has a concentration-dependent effect on pregnancy and birth rates [5]. However, Zavy et al. [6] and Wang et al. [7] reported that serum E2 on the day of hCG administration does not change the pregnancy rate. Based on these data, the importance of managing high E2 levels on the day of hCG administration remains controversial in terms of IVF outcomes. The objective of our study was to evaluate the effect of the hCG-day serum E2 level on neonatal birthweight after IVF-ET with $\mathrm{COH}$.

\section{Methods}

\section{Ethical approval}

This study was approved by the Institutional Ethics Committee of the First Hospital of Zhengzhou University.

\section{Patient selection}

This was a single-centre retrospective cohort study. Patients who were undergoing IVF with gonadotropin and a GnRH agonist for $\mathrm{COH}$ were enrolled from August 2009 to February 2017. Patients were excluded from the study if they experienced one of the following conditions: (1) moderate or severe ovarian hyper-stimulation syndrome (OHSS) during $\mathrm{COH}$; (2) pregnancy complications including gestational diabetes mellitus, hypertensive disorders and thyroid diseases; (3) vanishing twin syndrome, defined as a foetus with cardiac activity plus one or more gestational sacs or a foetus without cardiac activity [8]; or (4) loss to follow-up or loss of core data in our electronic database (e.g., E2 level on the trigger day).

In cases where a patient has given birth more than once, only the first live birth was included in the analysis during the study period. Ultimately, 3659 patients constituted our final study cohort. According to serum E2 levels on the day of hCG administration, the patients were categorized into six groups: group 1

Page $2 / 12$ 
(serum $E_{2}$ levels $\leq 1000 \mathrm{pg} / \mathrm{mL}, \mathrm{n}=230$ ), group 2 (serum $E_{2}$ levels 1001-2000 pg/mL, $\mathrm{n}=524$ ), group 3 (serum $\mathrm{E}_{2}$ levels 2001-3000 pg/mL, $\mathrm{n}=783$ ), group 4 (serum $E_{2}$ levels $3001-4000 \mathrm{pg} / \mathrm{mL}, \mathrm{n}=721$ ), group 5 (serum $\mathrm{E}_{2}$ levels $4001-5000 \mathrm{pg} / \mathrm{mL}, \mathrm{n}=548$ ), and group 6 (serum $\mathrm{E}_{2}$ levels $>5000 \mathrm{pg} / \mathrm{mL}$, $\mathrm{n}=852$ ) [9].

\section{Controlled hyper-stimulation induction and embryo transfer}

All patients received one of the following four controlled ovarian stimulation (COS) regimens, which have been described previously [10]: gonadotropinreleasing hormone (GnRH)-agonist short protocol, GnRH-antagonist, mild stimulation and progestin-primed ovarian stimulation (PPOS). The clinician selected the appropriate protocol for each patient on an individual basis according to the patient characteristics.

Pituitary suppression was achieved by injecting triptorelin acetate (Decapeptyl® $0.1 \mathrm{mg}$ [Ferring, Germany] or Diphereline ${ }^{\circledR} 0.1 / 3.75 \mathrm{mg}$ [Ipsen, France]) until the serum levels of E2, follicle-stimulating hormone (FSH) and luteinizing hormone (LH) were $<30 \mathrm{mIU} / \mathrm{mL},<5 \mathrm{mIU} / \mathrm{mL}$ and $<5 \mathrm{mIU} / \mathrm{mL}$, respectively. COS was initiated with several types of gonadotropin, namely, FSH (Gonal-F® 75 IU[Serono, Switzerland], Fostiman® 75 IU [IBSA, Switzerland],or Puregon $\circledast 50$ IU [N. V. Organon, Netherlands]) or FSH combined with LH (hMG®, Livzon, China). In general, the initial dose of gonadotropin was determined according to the individual's age, BMI, basal follicle-stimulating hormone (bFSH) level, and response to previous stimulation cycles, as well as whether polycystic ovary syndrome (PCOS) was present.

After stimulation for at least 4 days, the dose was adjusted according to the ovarian response, which was assessed using ultrasound and serum E2 measurements. When more than 3 follicles reached $17 \mathrm{~mm}$, hCG was injected, and oocytes were extracted $36-37 \mathrm{~h}$ later.

\section{Serum hormone measurements}

bFSH was measured on days 2-4 of the menstrual cycle and prior to the start of the IVF cycle. In each cycle, LH, E2 and P levels were measured during controlled ovulation induction (once every 2-4 days at the beginning, once every day in the late follicular stage, and once on the day of hCG injection), and the gonadotropin and gonadotropin levels of each group were compared. All blood samples were obtained in the fasting state, usually between 6:30 and 7:30 am. Throughout the study, we used a personal immune analyser (Roche Cobas e411; Roche Diagnostics, Mannheim, Germany) and the same analytical methods were used to measure all hormones.

\section{Data collection}

With a standardized questionnaire telephone survey, specially trained nurses in our department conducted surveys of couples during each pregnancy to collect information on pregnancy complications, pregnancy date, place of birth, delivery mode, sex of the new-born, gestational age, birth weight, and neonatal diseases. In cases where attempts to contact the couple failed, the local family planning service was contacted to collect data.

\section{Outcome measures}

The outcomes were birth weight indicators, including absolute birth weight and LBW. LBW was defined as birthweight <2500 g. Preterm birth (PTB) was defined as delivery before 37 weeks of gestation.

\section{Statistical analysis}

All continuous variables are expressed as means \pm SDs and categorical variables as frequencies or percentages. To examine significant differences among groups, the Mann-Whitney and chi-square tests were used for continuous variables and categorical variables, respectively. Multiple logistic regression models were applied to examine the relationship between serum E2 levels and neonatal birthweight. The relationship between the serum E2 level and neonatal birthweight was also explored using smoothing plots. A two-piecewise linear regression model was applied to investigate the threshold effect according to the smoothing plot. To quantify the strength of the association, unadjusted and adjusted odds ratios (ORs) and 95\% confidence intervals (Cis) were estimated and reported. In the multivariate adjusted logistic regression models, the following were considered confounders: maternal age (years); sterility classification; duration of infertility (years); maternal BMI (kg/m2); basal FSH (mlU/mL); basal E2 (pg/mL); basal LH (mlU/mL); previous IVF attempts; ovarian stimulation protocol; total hMG dose (IU); duration of stimulation (days); number of oocytes retrieved; number of viable embryos; number of embryos transferred; gestational age at delivery (weeks); and neonatal sex. Interaction and stratified analyses included maternal age (years), maternal BMI, previous IVF attempts, and number of embryos transferred. Receiver operator characteristics (ROC) curves were constructed to identify the E2 threshold, and the corresponding area under the curve (AUC) was calculated.

Data were analysed with the use of the statistical packages R (The R Foundation; http://www.r-project.org; version 3.4.3) and EmpowerStats (www.empowerstats.com; X\&Y Solutions, Inc., Boston, MA).

\section{Results}

Overall, 3659 singleton live births from IVF cycles were included in this retrospective study. The numbers of patients with a peak serum E2 level of <1000, 1000-1999, 2000-2999, 3000-3999, 4000-4999 and $\geq 5000$ pg/mL were 230 (6.3\%), 524 (14.3\%), 783 (21.4\%), 721 (19.7\%), 548 (15.0\%) and 852 (23.3\%), respectively.

Table 1 shows the baseline demographic and periodic characteristics of the study cohort. Briefly, there were no significant differences in average age or BMI between the six groups, the average age and BMI of the first group were the highest, at $32.1 \pm 4.4$ years and $23.2 \pm 3.2 \mathrm{~kg} / \mathrm{m} 2$, respectively. The lowest hMG dose was $1939.5 \pm 849.9 \mathrm{IU}$ in the sixth group with the highest E2 level on the injection day of hCG. This may indicate that the low injection amount of hCG 
during downregulation may lead to a high E2 level on the hCG injection day, and previous research supports this finding [11]. The majority of patients underwent double and cleavage-stage embryo transfer

Table 1. Baseline characteristics of the study participants

\begin{tabular}{|c|c|c|c|c|c|c|}
\hline \multirow[b]{2}{*}{ Baseline characteristics } & \multicolumn{6}{|c|}{ Oestradiol level $(\mathrm{pg} / \mathrm{mL})$ on the day of trigger } \\
\hline & $\leq 1000$ & $>1000, \leq 2000$ & $>2000, \leq 3000$ & $>3000, \leq 4000$ & $>4000, \leq 5000$ & $>5000$ \\
\hline $\mathbf{N}$ & $230(6.3 \%)$ & $524(14.3 \%)$ & $783(21.4 \%)$ & $721(19.7 \%)$ & $548(15.0 \%)$ & $852(23.3 \%)$ \\
\hline Maternal age (years) & $32.1 \pm 4.4$ & $31.8 \pm 4.7$ & $31.3 \pm 4.4$ & $30.9 \pm 4.4$ & $30.5 \pm 4.6$ & $30.1 \pm 4.2$ \\
\hline Duration of infertility (years) & $4.2 \pm 3.1$ & $4.3 \pm 3.3$ & $4.1 \pm 3.0$ & $4.0 \pm 2.9$ & $4.0 \pm 3.1$ & $4.1 \pm 3.0$ \\
\hline Maternal BMI (kg/m2) & $23.2 \pm 3.2$ & $23.2 \pm 3.2$ & $22.8 \pm 3.4$ & $22.3 \pm 3.0$ & $22.0 \pm 2.9$ & $21.9 \pm 2.9$ \\
\hline Basal FSH (mlU/mL) & $7.9 \pm 4.8$ & $7.7 \pm 3.8$ & $7.1 \pm 2.2$ & $7.1 \pm 2.4$ & $6.9 \pm 2.0$ & $6.9 \pm 2.3$ \\
\hline Basal E2 (pg/mL) & $49.6 \pm 59.2$ & $47.2 \pm 44.5$ & $45.1 \pm 38.7$ & $47.8 \pm 51.7$ & $46.7 \pm 45.2$ & $45.3 \pm 27.2$ \\
\hline Basal LH (mlU/mL) & $5.3 \pm 4.0$ & $4.9 \pm 3.4$ & $4.9 \pm 2.8$ & $5.2 \pm 3.0$ & $5.3 \pm 2.9$ & $5.8 \pm 3.6$ \\
\hline Total hMG dose (IU) & $2751.3 \pm 1308.9$ & $2761.1 \pm 1142.7$ & $2593.5 \pm 1014.4$ & $2360.5 \pm 972.7$ & $2112.3 \pm 848.9$ & $1939.5 \pm 849.9$ \\
\hline Duration of stimulation (days) & $10.8 \pm 5.4$ & $11.4 \pm 4.7$ & $11.3 \pm 4.9$ & $11.0 \pm 4.8$ & $10.3 \pm 4.9$ & $9.8 \pm 5.2$ \\
\hline number of oocytes retrieved & $5.9 \pm 5.3$ & $6.4 \pm 4.1$ & $8.4 \pm 4.6$ & $9.1 \pm 4.7$ & $10.7 \pm 5.2$ & $12.6 \pm 5.4$ \\
\hline delivery gestational age (weeks) & $38.6 \pm 1.5$ & $38.7 \pm 1.5$ & $38.9 \pm 1.5$ & $38.8 \pm 1.5$ & $38.9 \pm 1.5$ & $39.0 \pm 1.4$ \\
\hline Birthweight (g) & $3496.7 \pm 721.9$ & $3456.8 \pm 657.1$ & $3451.1 \pm 557.6$ & $3443.3 \pm 620.8$ & $3407.8 \pm 583.4$ & $3496.9 \pm 724.0$ \\
\hline \multicolumn{7}{|l|}{ Sterility classification } \\
\hline Primary infertility & $112(48.7 \%)$ & $254(48.5 \%)$ & $361(46.1 \%)$ & $371(51.5 \%)$ & $279(50.9 \%)$ & $439(51.5 \%)$ \\
\hline Second infertility & $118(51.3 \%)$ & $270(51.5 \%)$ & $422(53.9 \%)$ & $350(48.5 \%)$ & $269(49.1 \%)$ & $413(48.5 \%)$ \\
\hline \multicolumn{7}{|l|}{ Previous IVF attempts } \\
\hline 0 & $178(77.4 \%)$ & $439(83.8 \%)$ & $659(84.2 \%)$ & $630(87.4 \%)$ & $494(90.1 \%)$ & $777(91.2 \%)$ \\
\hline 1 & $36(15.7 \%)$ & $60(11.5 \%)$ & $99(12.6 \%)$ & $76(10.5 \%)$ & $47(8.6 \%)$ & $60(7.0 \%)$ \\
\hline 2 & $15(6.5 \%)$ & $17(3.2 \%)$ & $17(2.2 \%)$ & $11(1.5 \%)$ & $5(0.9 \%)$ & $12(1.4 \%)$ \\
\hline 3 & $1(0.4 \%)$ & $6(1.1 \%)$ & $6(0.8 \%)$ & $4(0.6 \%)$ & $1(0.2 \%)$ & $3(0.4 \%)$ \\
\hline 4 & $0(0.0 \%)$ & $1(0.2 \%)$ & $1(0.1 \%)$ & $0(0.0 \%)$ & $1(0.2 \%)$ & $0(0.0 \%)$ \\
\hline 6 & $0(0.0 \%)$ & $1(0.2 \%)$ & $1(0.1 \%)$ & $0(0.0 \%)$ & $0(0.0 \%)$ & $0(0.0 \%)$ \\
\hline \multicolumn{7}{|l|}{ Ovarian stimulation protocol } \\
\hline GnRH-a long protocol & $102(44.3 \%)$ & $238(45.4 \%)$ & $366(46.7 \%)$ & $474(65.7 \%)$ & $416(75.9 \%)$ & $718(84.3 \%)$ \\
\hline GnRH-a prolonged (modified) protocol & $111(48.3 \%)$ & $278(53.1 \%)$ & $413(52.7 \%)$ & $247(34.3 \%)$ & $131(23.9 \%)$ & $130(15.3 \%)$ \\
\hline mild stimulation & $17(7.4 \%)$ & $8(1.5 \%)$ & $2(0.3 \%)$ & $0(0.0 \%)$ & $1(0.2 \%)$ & $1(0.1 \%)$ \\
\hline GnRH-agonist short protocol & $0(0.0 \%)$ & $0(0.0 \%)$ & $2(0.3 \%)$ & $0(0.0 \%)$ & $0(0.0 \%)$ & $3(0.4 \%)$ \\
\hline \multicolumn{7}{|l|}{ Number of embryos transfer } \\
\hline 1 & $55(23.9 \%)$ & $52(9.9 \%)$ & $30(3.8 \%)$ & $17(2.4 \%)$ & $9(1.6 \%)$ & $20(2.3 \%)$ \\
\hline 2 & $169(73.5 \%)$ & $446(85.1 \%)$ & $687(87.7 \%)$ & $648(89.9 \%)$ & $493(90.0 \%)$ & $764(89.7 \%)$ \\
\hline 3 & $6(2.6 \%)$ & $26(5.0 \%)$ & $66(8.4 \%)$ & $56(7.8 \%)$ & $46(8.4 \%)$ & $68(8.0 \%)$ \\
\hline
\end{tabular}

NOTE: Data are presented as the mean \pm SD or number (percentage). BMI: Body mass index; FSH: Follicle-stimulating hormone; E2: Oestradiol; LH: Luteinizing hormone; IVF: In vitro fertilization

Table 2 shows the single-factor analysis of neonatal birthweight. As shown, maternal BMI ( $\mathrm{kg} / \mathrm{m} 2)$, basal LH (mIU/mL), and gestational age at delivery (weeks) were strongly correlated with neonatal birthweight (all P-values $<0.001$ ). Bacârea et al. [12] found a positive correlation between maternal BMI and neonatal birth weight [12], which is consistent with our data analysis. The increase in maternal gestational age is positively correlated with neonatal weight, which is consistent with normal physiological dynamics. However, there seems to be no similar study on whether maternal serum basal LH is associated with neonatal birth weight, so further statistical analysis of the clinical sample data or basic experiments are needed to verify this. In addition, the effect of different ovulation induction schemes for fresh cycles on new-born birthweight was also correlated, the specific mechanism remains to be explored. 
Table 2. Single-factor analysis of neonatal birthweight

\begin{tabular}{|c|c|c|c|c|c|}
\hline \multirow[t]{2}{*}{ Comparison } & \multirow[t]{2}{*}{ Statistics } & \multirow[t]{2}{*}{ Sig. } & \multirow[t]{2}{*}{$\operatorname{Exp}(B)$} & \multicolumn{2}{|l|}{$95 \% \mathrm{Cl}$} \\
\hline & & & & Lower & Upper \\
\hline Maternal age (years) & $30.9 \pm 4.5$ & 0.071 & 4.3 & -0.4 & 8.9 \\
\hline \multicolumn{6}{|l|}{ Sterility classification } \\
\hline Primary infertility & $1816(49.6 \%)$ & 0 & & & \\
\hline Second infertility & $1842(50.4 \%)$ & 0.458 & 15.7 & -25.8 & 57.2 \\
\hline Duration of infertility (years) & $4.1 \pm 3.0$ & 0.065 & 6.5 & -0.4 & 13.3 \\
\hline \multicolumn{6}{|l|}{ Previous IVF attempts } \\
\hline 0 & 3177 (86.9\%) & 0 & & & \\
\hline 1 & $378(10.3 \%)$ & 0.675 & 14.6 & -53.7 & 83.0 \\
\hline 2 & $77(2.1 \%)$ & 0.898 & -9.5 & -154.3 & 135.4 \\
\hline$\geq 3$ & $26(0.7 \%)$ & 0.628 & 61.2 & -186.1 & 308.6 \\
\hline Maternal BMI (kg/m2) & $22.5 \pm 3.1$ & $<0.001$ & 20.3 & 13.7 & 27.0 \\
\hline Basal FSH (mIU/mL) & $7.2 \pm 2.8$ & 0.101 & 6.3 & -1.2 & 13.8 \\
\hline Basal E2 (pg/mL) & $46.5 \pm 42.8$ & 0.673 & -0.1 & -0.6 & 0.4 \\
\hline Basal LH (mlU/mL) & $5.2 \pm 3.2$ & $<0.001$ & 11.2 & 4.8 & 17.7 \\
\hline \multicolumn{6}{|l|}{ Ovarian stimulation protocol } \\
\hline GnRH-a long protocol & $2314(63.3 \%)$ & 0 & & & \\
\hline GnRH-a prolonged (modified) protocol & $1310(35.8 \%)$ & $<0.001$ & -99.3 & -142.6 & -56.0 \\
\hline Mild stimulation & $29(0.8 \%)$ & 0.285 & -127.6 & -361.5 & 106.3 \\
\hline GnRH-agonist short protocol & $5(0.1 \%)$ & 0.018 & 676.9 & 116.5 & 1237.3 \\
\hline Total hMG dose (IU) & $2357.1 \pm 1034.9$ & 0.069 & 0.0 & -0.0 & 0.0 \\
\hline Duration of stimulation (days) & $10.7 \pm 5.0$ & 0.111 & -3.4 & -7.5 & 0.8 \\
\hline Serum E2 (pg/ml), hCG day & $3736.4 \pm 2123.3$ & 0.154 & 0.0 & -0.0 & 0.0 \\
\hline Number of oocytes retrieved & $9.4 \pm 5.4$ & 0.369 & -1.8 & -5.6 & 2.1 \\
\hline \multicolumn{6}{|l|}{ Number of embryos transferred } \\
\hline 1 & $183(5.0 \%)$ & 0 & & & \\
\hline 2 & 3207 (87.7\%) & 0.279 & -52.6 & -147.9 & 42.7 \\
\hline 3 & $268(7.3 \%)$ & 0.130 & 93.0 & -27.3 & 213.2 \\
\hline Gestational age at delivery (weeks) & $38.9 \pm 1.5$ & $<0.001$ & 117.7 & 104.2 & 131.2 \\
\hline
\end{tabular}

Outcome: Birthweight (g)

Exposure: Maternal age (years); Sterility classification; Duration of infertility (years); Previous IVF attempts NEW; Maternal BMI (kg/m2); Basal FSH (mIU/mL); Basal E2 (pg/mL); Basal LH (mlU/mL); Ovarian stimulation protocol; Total hMG dose (IU); Duration of stimulation (days); Serum E2 (pg/ml), hCG day; Number of oocytes retrieved; Number of viable embryos; Number of embryos transferred; Gestational age at delivery (weeks);

Serum E2 (pg/ml), hCG day; Oestradiol level $(\mathrm{pg} / \mathrm{mL})$ on trigger day

Table 3 shows the neonatal outcomes grouped by oestradiol level on the trigger day. The average gestational age at delivery in the group 6 (serum E2 levels > $5000 \mathrm{pg} / \mathrm{mL}$ ) was $39.0 \pm 1.4$ weeks $(P=0.010)$. The lowest mean neonatal birthweight in group 5 (serum E2 levels $4001-5000 \mathrm{pg} / \mathrm{mL}$ ) was $3407.8 \pm 583.4 \mathrm{~g}$ $(\mathrm{P}=0.167)$. This is consistent with the line graph of the mean after adjustments for variables in Figure 1. The mean new-born birthweight in the group 5 showed a slight downward trend. In addition, the LBW rates in the six groups were $3.0 \%, 2.9 \%, 1.9 \%, 2.9 \%, 2.9 \%$, and $2.0 \%$ ( $P=0.629)$, respectively. There were no statistically significant differences in the incidences of neonatal LBW among the six groups $(\mathrm{P}=0.629)$, which is controversial with previous reports by Pereira et al. [13], and their study shows that a peak E2 level $>3,069.2 \mathrm{pg} / \mathrm{mL}$ is associated with increased odds of LBW term singletons after fresh IVF-ET cycles[13]. In view of this contradiction, we continued to carry out data analysis.

Table 3. Neonatal outcomes grouped by oestradiol level on trigger day

Page 5/12 


\begin{tabular}{|c|c|c|c|c|c|c|c|}
\hline \multirow[b]{2}{*}{ Baseline characteristics } & \multicolumn{7}{|c|}{ Grouped by oestradiol level $(\mathrm{pg} / \mathrm{ml})$ on the day of trigger } \\
\hline & $<=1000$ & $>1000,<=2000$ & $>2000,<=3000$ & $>3000,<=4000$ & $>4000,<=5000$ & $>5000$ & $P$-value \\
\hline $\mathbf{N}$ & 230 & 524 & 783 & 721 & 548 & 852 & \\
\hline Delivery gestational age (weeks) & $38.6 \pm 1.5$ & $38.7 \pm 1.5$ & $38.9 \pm 1.5$ & $38.8 \pm 1.5$ & $38.9 \pm 1.5$ & $39.0 \pm 1.4$ & $0.010^{*}$ \\
\hline Preterm birth, n (\%) & $13(5.7 \%)$ & $29(5.6 \%)$ & $35(4.5 \%)$ & $36(5.0 \%)$ & $20(3.7 \%)$ & $30(3.5 \%)$ & 0.377 \\
\hline Birthweight (g) & $3496.7 \pm 721.9$ & $3456.8 \pm 657.1$ & $3451.1 \pm 557.6$ & $3443.3 \pm 620.8$ & $3407.8 \pm 583.4$ & $3496.9 \pm 724.0$ & 0.167 \\
\hline Sex & & & & & & & 0.783 \\
\hline Male & $124(53.9 \%)$ & $257(49.0 \%)$ & $400(51.1 \%)$ & 375 (52.0\%) & $290(52.9 \%)$ & $444(52.1 \%)$ & \\
\hline Female & $106(46.1 \%)$ & 267 (51.0\%) & 383 (48.9\%) & $346(48.0 \%)$ & 258 (47.1\%) & 408 (47.9\%) & \\
\hline Birthweight (g) group & & & & & & & 0.629 \\
\hline$\geq 2500$ (Non-LBW) & $223(97.0 \%)$ & $509(97.1 \%)$ & $768(98.1 \%)$ & 700 (97.1\%) & $532(97.1 \%)$ & $835(98.0 \%)$ & \\
\hline <2500 (LBW) & $7(3.0 \%)$ & $15(2.9 \%)$ & $15(1.9 \%)$ & $21(2.9 \%)$ & $16(2.9 \%)$ & $17(2.0 \%)$ & \\
\hline
\end{tabular}

Data are presented as the mean \pm SD or number (percentage).

LBW: low birth weight.

Non-LBW: non-low birth weight.

$P$-values $<0.05$ indicate significant differences and are bolded.

In Table 4, we conducted a stratified analysis of neonatal birthweight. The statistical results showed that the previous conclusions were stable and reliable, and there was no statistically significant difference in neonatal weight among the six groups. At the same time, in Table 5, we adjusted for confounders and conducted a multiple regression analysis of intergroup LBW outcomes, the results of which supported our conclusions above.

Table 4. Geometric mean values for neonatal birthweight (g) across categories 


\begin{tabular}{|c|c|c|c|c|c|c|c|c|c|c|c|c|c|c|c|}
\hline \multirow{4}{*}{$\begin{array}{l}\text { Y= } \\
\text { Birthweight } \\
\text { (g) }\end{array}$} & \multicolumn{15}{|c|}{ Grouped by oestradiol level $(\mathrm{pg} / \mathrm{ml})$ on the trigger day } \\
\hline & \multirow[t]{3}{*}{$\mathbf{N}$} & \multirow[t]{3}{*}{$\leq 1000$} & \multicolumn{4}{|c|}{$>1000, \leq 2000$} & \multicolumn{4}{|c|}{$>2000, \leq 3000$} & \multicolumn{4}{|c|}{$>3000, \leq 4000$} & \multirow{3}{*}{$\begin{array}{l}>40 \\
\text { Sig. }\end{array}$} \\
\hline & & & \multirow[t]{2}{*}{ Sig. } & \multirow[t]{2}{*}{$\operatorname{Exp}(B)$} & \multicolumn{2}{|l|}{$95 \% \mathrm{Cl}$} & \multirow[t]{2}{*}{ Sig. } & \multirow[t]{2}{*}{$\operatorname{Exp}(B)$} & \multicolumn{2}{|l|}{$95 \% \mathrm{Cl}$} & \multirow[t]{2}{*}{ Sig. } & \multirow[t]{2}{*}{$\operatorname{Exp}(B)$} & \multicolumn{2}{|l|}{$95 \% \mathrm{Cl}$} & \\
\hline & & & & & Lower & Upper & & & Lower & Upper & & & Lower & Upper & \\
\hline \multicolumn{16}{|l|}{$\begin{array}{l}\text { Matemal } \\
\text { age (years) } \\
\text { group }\end{array}$} \\
\hline $\begin{array}{l}\text { Group } 1 \\
(<26.5)\end{array}$ & 887 & 0 & 0.286 & 121.7 & -101.7 & 345.0 & 0.763 & 33.0 & -181.1 & 247.2 & 0.288 & 115.2 & -96.9 & 327.2 & 0.43 \\
\hline $\begin{array}{l}\text { Group } 2 \\
(\geq 26.5 \\
<29.1)\end{array}$ & 909 & 0 & $0.007 *$ & -274.1 & -471.1 & -77.1 & $0.024 *$ & -211.3 & -393.9 & -28.7 & $0.012^{*}$ & -237.6 & -423.1 & -52.1 & 0.01 \\
\hline $\begin{array}{l}\text { Group } 3 \\
(\geq 29.1 \\
<31.7)\end{array}$ & 816 & 0 & 0.185 & 143.0 & -68.1 & 354.0 & $0.077^{*}$ & 179.0 & -19.2 & 377.1 & 0.407 & 84.8 & -115.4 & 284.9 & 0.89 \\
\hline $\begin{array}{l}\text { Group } 4 \\
(\geq 31.7)\end{array}$ & 1046 & 0 & 0.582 & -50.0 & -228.0 & 128.0 & 0.367 & -79.0 & -250.7 & 92.6 & 0.439 & -69.7 & -246.1 & 106.7 & 0.31 \\
\hline \multicolumn{16}{|l|}{$\begin{array}{l}\text { Matemal } \\
\text { BMl } \\
\text { (kg/m2) } \\
\text { group }\end{array}$} \\
\hline $\begin{array}{l}\text { Group } 1 \\
(<15.75)\end{array}$ & 527 & 0 & 0.052 & -288.7 & -579.3 & 1.9 & $0.022^{*}$ & -303.3 & -562.3 & -44.3 & $0.007 *$ & -355.5 & -613.1 & -97.8 & 0.00 \\
\hline $\begin{array}{l}\text { Group 2 } \\
(\geq 15.75 \\
<21.25)\end{array}$ & 1045 & 0 & 0.602 & -53.1 & -252.4 & 146.2 & 0.284 & -105.1 & -297.5 & 87.3 & 0.761 & -29.5 & -219.5 & 160.4 & 0.65 \\
\hline $\begin{array}{l}\text { Group } 3 \\
(\geq 21.25 \\
<26.75)\end{array}$ & 897 & 0 & 0.750 & 33.6 & -173.2 & 240.5 & 0.556 & 58.4 & -135.9 & 252.7 & 0.950 & 6.3 & -192.3 & 204.8 & 0.54 \\
\hline $\begin{array}{l}\text { Group 4 } \\
(\geq 26.75)\end{array}$ & 1189 & 0 & 0.981 & -1.9 & -159.2 & 155.4 & 0.765 & 23.0 & -127.7 & 173.8 & 0.690 & 31.7 & -124.1 & 187.6 & 0.28 \\
\hline \multicolumn{16}{|l|}{ Sex } \\
\hline Male & 1890 & 0 & 0.529 & -42.9 & -176.7 & 90.9 & 0.411 & -52.8 & -178.6 & 73.0 & 0.128 & -98.5 & -225.2 & 28.3 & 0.04 \\
\hline Female & 1768 & 0 & 0.745 & -24.4 & -171.9 & 123.0 & 0.672 & -30.4 & -171.4 & 110.5 & 0.992 & 0.8 & -141.8 & 143.4 & 0.62 \\
\hline \multicolumn{16}{|l|}{$\begin{array}{l}\text { Number of } \\
\text { embryos } \\
\text { transferred }\end{array}$} \\
\hline 1 & 183 & 0 & $0.098 *$ & -229.3 & -499.3 & 40.6 & 0.411 & -133.2 & -449.9 & 183.6 & 0.191 & -259.5 & -646.8 & 127.8 & 0.72 \\
\hline 2 & 3207 & 0 & 0.904 & -6.6 & -113.1 & 100.0 & 0.797 & -13.3 & -114.6 & 88.0 & 0.659 & -23.0 & -124.9 & 78.9 & 0.40 \\
\hline 3 & 268 & 0 & 0.980 & 10.6 & -832.8 & 853.9 & 0.585 & -221.4 & -1015.4 & 572.6 & 0.692 & -161.6 & -961.5 & 638.3 & 0.33 \\
\hline
\end{tabular}

$P$-values $<0.05$ indicate significant differences and are bolded.

Table 5. Multivariable logistic model for all patients

\begin{tabular}{|c|c|c|c|c|c|c|c|c|c|c|c|c|}
\hline \multirow{3}{*}{$\begin{array}{l}\text { Oestradiol level }(\mathrm{pg} / \mathrm{ml}) \text { on the trigger } \\
\text { day }\end{array}$} & \multicolumn{4}{|c|}{ Non-adjusted } & \multicolumn{4}{|c|}{ Adjustment I } & \multicolumn{4}{|c|}{ Adjustment II } \\
\hline & \multirow[t]{2}{*}{ Sig. } & \multirow[t]{2}{*}{$\operatorname{Exp}(B)$} & \multicolumn{2}{|l|}{$95 \% \mathrm{Cl}$} & \multirow[t]{2}{*}{ Sig. } & \multirow[t]{2}{*}{$\operatorname{Exp}(B)$} & \multicolumn{2}{|l|}{$95 \% \mathrm{Cl}$} & \multirow[t]{2}{*}{ Sig. } & \multirow[t]{2}{*}{$\operatorname{Exp}(B)$} & \multicolumn{2}{|l|}{$95 \% \mathrm{Cl}$} \\
\hline & & & Lower & Upper & & & Lower & Upper & & & Lower & Upper \\
\hline$\leq 1000$ & - & 1.0 & - & - & - & 1.0 & - & - & - & 1.0 & - & - \\
\hline$>1000, \leq 2000$ & 0.892 & 0.9 & 0.4 & 2.3 & 0.905 & 0.9 & 0.4 & 2.4 & 0.905 & 1.1 & 0.3 & 3.3 \\
\hline$>2000, \leq 3000$ & 0.307 & 0.6 & 0.3 & 1.5 & 0.325 & 0.6 & 0.3 & 1.6 & 0.615 & 0.7 & 0.2 & 2.4 \\
\hline$>3000, \leq 4000$ & 0.919 & 1.0 & 0.4 & 2.3 & 0.949 & 1.0 & 0.4 & 2.3 & 0.663 & 1.3 & 0.4 & 4.1 \\
\hline$>4000, \leq 5000$ & 0.926 & 1.0 & 0.4 & 2.4 & 0.968 & 1.0 & 0.4 & 2.4 & 0.437 & 1.6 & 0.5 & 5.5 \\
\hline$>5000$ & 0.342 & 0.6 & 0.3 & 1.6 & 0.383 & 0.7 & 0.3 & 1.6 & 0.625 & 1.4 & 0.4 & 4.8 \\
\hline
\end{tabular}

Non-adjusted model: no adjustments 
Adjustment model l: adjusted for maternal age (years); sterility classification; duration of infertility (years); and maternal BMI (kg/m2)

Adjustment model II: adjusted for maternal age (years); sterility classification; duration of infertility (years); maternal BMI (kg/m2); basal FSH (mIU/mL); basal E2 (pg/mL); basal LH (mIU/mL); previous IVF attempts; ovarian stimulation protocol; total hMG dose (IU); duration of stimulation (days); number of oocytes retrieved; number of viable embryos; number of embryos transferred; gestational age at delivery (weeks); and neonatal sex

To explore the relationship between serum E2 level and neonatal weight on hCG day and whether there is a threshold effect, the analyses shown in Figures 2 and 3 were performed. Figure 2 shows a nonlinear relationship between serum E2 levels and neonatal birthweight after adjusting for maternal age, maternal $\mathrm{BMI}$, number of embryos transferred, and neonatal sex. In Figure 3, the ROC curve is presented to evaluate the ability of peak E2 measurements to predict LBW. The best threshold is $2062 \mathrm{pg} / \mathrm{ml}$ with a sensitivity of $28.6 \%$, a specificity of $78.1 \%$, and an AUC of 0.518 . Such a small AUC value does not have sufficient strength as evidence.

\section{Discussion}

The results of this retrospective cohort study showed that serum E2 peak levels during ovarian stimulation were not associated with birthweight during IVF cycles. In addition, there was no association between higher E2 levels and increased LBW risk. Our observations suggest that the hyper-oestrogenic milieu during COS does not seem to have adverse effects on the birthweight of offspring after IVF.

A supraphysiologic E2 level is inevitable during $\mathrm{COH}$, and its effect on the outcome of IVF-ET has been controversial. Oestrogen and its receptors are a major factor in improving endometrial receptivity to initiate embryo implantation [14]. There is increasing evidence that hyper-physiological E2 levels during COS may lead to decreased endometrial and sub-endometrial blood flow [15]. These harmful effects not only impair early embryo adhesion and implantation [16] but also harm placental formation and subsequent foetal growth [17]. The high oestradiol group had a high incidence of pregnancy complications associated with placental abnormalities, including foetal growth restriction, pregnancy-related hypertension, and abnormal placental implantation [17]. Therefore, in clinical work, the possible risk of OHSS and the pregnancy complications associated with placental abnormalities should be considered when deciding on embryo transfer in high responders. However, the results of our retrospective cohort study showed that serum E2 peak levels during ovarian stimulation were not associated with birthweight during IVF cycles. Zavy et al. [6] and Wang et al. [7] reported that an elevated serum E2 level on the day of hCG injection does not change the pregnancy rate, indirectly confirming our conclusion.

Recently, Imudia et al. [18] found that the fertilization rate of normal oocytes, the embryo development rate and the positive rate for pregnancy showed an overall decreasing trend with increasing oestradiol level[18]. However, Imudia et al. [18] found that a serum E2 level that exceeds the 90th percentile on hCG injection day is associated with a decreased oocyte fertilization rate but that this level does not affect embryo development, the implantation rate, the clinical pregnancy rate or spontaneous abortion rate[18]. Chen et al. [19] showed that peak E2 levels do not adversely affect treatment outcomes. Wu et al. [20] suggested that a single-day high E2 concentration on the hCG trigger day would not affect the pregnancy outcome; however, when combined with a premature progesterone elevation, this high E2 concentration may have adverse effects on pregnancy outcomes. For these patients, frozen-thawed embryo transfer (FET) should be recommended to improve pregnancy outcomes [20]. Other studies [20-22] have reported similar results in response to the view that elevated E2 levels do not have any harmful effects on pregnancy rates.

Understanding and managing patients with ovarian hyper-responsiveness in clinical practice can be a challenge for physicians. In our study, patients with OHSS were excluded. In addition to OHSS, hyper-physiological levels of oestradiol are well documented and may cause endometria-embryo dys-synchrony, which may have a negative impact on pregnancy rates. After controlling for embryo quality, Zavy et al. [6] showed that the live birth rate is not affected by the increase in E2 level on hCG injection day, and this conclusion is consistent with the actual practice in our clinical work, as well as our statistical conclusion. In our clinical work, high-quality embryos are given priority for transplantation. This may lead to a statistical bias in selectivity. However, this is more in line with the actual situation in clinical work. Our study showed that, after controlling for embryo quality, an increase in oestradiol levels on the day of hCG injection was neither directly harmful nor beneficial to neonatal birthweight. At the same time, our data suggest that high-quality embryos may be able to tolerate suboptimal uterine environments that have been exposed to hyper-physiological levels of oestradiol. Therefore, the quality control of embryos is a very important part of IVF.

\section{Conclusion}

In clinical work, controlling the quality of transplanted embryos is very important. We did not detect an association between peak serum E2 level during ovarian stimulation and neonatal birthweight IVF-ET cycles. This finding provides reliable information not only for patients with high ovarian response but also for clinicians in choosing an appropriate $\mathrm{COH}$ regime.

\section{Abbreviations}

hCG: human chorionic gonadotrophin

IVF-ET: in vitro fertilization-embryo transfer

BMI: Body mass index

FSH: Follicle-stimulating hormone 
E2: Oestradiol

LH: Luteinizing hormone

IVF: In vitro fertilization

LBW: low birth weight

Non-LBW: non-low birth weight.

OHSS: Ovarian Hyper-stimulation Syndrome

\section{Declarations}

\section{Ethics approval and consent to participate}

This study was approved by the Institutional Ethics Committee of the First Hospital of Zhengzhou University. All patients signed informed consent forms.

\section{Consent for publication}

Not applicable

\section{Availability of data and materials}

Not applicable

\section{Competing interests}

We declare that we have no competing interests.

\section{Funding}

Not applicable

\section{Authors' contributions}

Yu Liu analyzed and interpreted the patient data and was a major contributor in writing the manuscript. All authors read and approved the final manuscript.

\section{Acknowledgements}

Not applicable

\section{Authors' information}

\section{References}

1. Steptoe PC, Edwards RG. Birth after the reimplantation of a human embryo. Lancet. 1978;2:366.

2. Adamson GD, de Mouzon J, Chambers GM, Zegers-Hochschild F, Mansour R, Ishihara O, et al. International Committee for Monitoring Assisted Reproductive Technology: world report on assisted reproductive technology, 2011. Fertil Steril. 2018;110:1067-80.

3. Qin JB, Sheng XQ, Wang H, Chen GC, Yang J, Yu H, et al. Worldwide prevalence of adverse pregnancy outcomes associated with in vitro fertilization/intracytoplasmic sperm injection among multiple births: a systematic review and meta-analysis based on cohort studies. Arch Gynecol Obstet. 2017;295:577-97.

4. Berntsen S, Soderstrom-Anttila V, Wennerholm UB, Laivuori H, Loft A, Oldereid NB, et al. The health of children conceived by ART: 'the chicken or the egg?'. Hum Reprod Update. 2019;25:137-58.

5. Steward RG, Zhang CE, Shah AA, Yeh JS, Chen C, Li YJ, et al. High peak estradiol predicts higher miscarriage and lower live birth rates in high responders triggered with a GnRH Agonist in IVF/ICSI cycles. J Reprod Med. 2015;60:463-70.

6. Zavy MT, Craig LB, Wild RA, Kahn SN, O'Leary D, Hansen KR. In high responding patients undergoing an initial IVF cycle, elevated estradiol on the day of hCG has no effect on live birth rate. Reprod Biol Endocrinol. 2014;12:119.

7. Wang M, Hao C, Bao H, Huang X, Liu Z, Zhang W, et al. Effect of elevated estradiol levels on the hCG administration day on IVF pregnancy and birth outcomes in the long GnRH-agonist protocol: analysis of 3393 cycles. Arch Gynecol Obstet. 2017;295:407-14.

8. Magnus MC, Ghaderi S, Morken NH, Magnus P, Bente Romundstad L, Skjaerven R, et al. Vanishing twin syndrome among ART singletons and pregnancy outcomes. Hum Reprod. 2017;32:2298-304.

9. Huang J, Lu X, Lin J, Chen Q, Gao H, Lyu Q, et al. Association between peak serum estradiol level during controlled ovarian stimulation and neonatal birthweight in freeze-all cycles: a retrospective study of 8501 singleton live births. Hum Reprod. 2020;35:424-33. 
10. Chen H, Wang Y, Lyu Q, Ai A, Fu Y, Tian H, et al. Comparison of live-birth defects after luteal-phase ovarian stimulation vs. conventional ovarian stimulation for in vitro fertilization and vitrified embryo transfer cycles. Fertil Steril. 2015;103:1194-201.e2.

11. Elnashar AM. Progesterone rise on the day of HCG administration (premature luteinization) in IVF: an overdue update. J Assist Reprod Genet. 2010;27:149-55.

12. Bacarea A, Bacarea VC, Tarcea M. The relation between prepregnancy maternal body mass index and total gestational weight gain with the characteristics of the newborns. J Matern Fetal Neonatal Med. 2020:1-6. doi:10.1080/14767058.2020.1818205.

13. Pereira N, Reichman DE, Goldschlag DE, Lekovich JP, Rosenwaks Z. Impact of elevated peak serum estradiol levels during controlled ovarian hyperstimulation on the birth weight of term singletons from fresh IVF-ET cycles. J Assist Reprod Genet. 2015;32:527-32.

14. Cai H, Zhu XX, Li ZF, Zhu YP, Lang JH. MicroRNA dysregulation and steroid hormone receptor expression in uterine tissues of rats with endometriosis during the implantation window. Chin Med J (Engl). 2018;131:2193-204.

15. Ng EH, Chan CC, Tang OS, Yeung WS, Ho PC. Comparison of endometrial and subendometrial blood flow measured by three-dimensional power Doppler ultrasound between stimulated and natural cycles in the same patients. Hum Reprod. 2004;19:2385-90.

16. Arslan M, Bocca S, Arslan EO, Duran HE, Stadtmauer L, Oehninger S. Cumulative exposure to high estradiol levels during the follicular phase of IVF cycles negatively affects implantation. J Assist Reprod Genet. 2007;24:111-7.

17. Farhi J, Ben-Haroush A, Andrawus N, Pinkas H, Sapir O, Fisch B, et al. High serum oestradiol concentrations in IVF cycles increase the risk of pregnancy complications related to abnormal placentation. Reprod Biomed Online. 2010;21:331-7.

18. Imudia AN, Goldman RH, Awonuga AO, Wright DL, Styer AK, Toth TL. The impact of supraphysiologic serum estradiol levels on peri-implantation embryo development and early pregnancy outcome following in vitro fertilization cycles. J Assist Reprod Genet. 2014;31:65-71.

19. Chen $\mathrm{CH}$, Zhang X, Barnes R, Confino E, Milad M, Puscheck E, et al. Relationship between peak serum estradiol levels and treatment outcome in in vitro fertilization cycles after embryo transfer on day 3 or day 5. Fertil Steril. 2003;80:75-9.

20. Wu Z, Li R, Ma Y, Deng B, Zhang X, Meng Y, et al. Effect of HCG-day serum progesterone and oestradiol concentrations on pregnancy outcomes in GnRH agonist cycles. Reprod Biomed Online. 2012;24:511-20.

21. Kyrou D, Popovic-Todorovic B, Fatemi HM, Bourgain C, Haentjens P, Van Landuyt L, et al. Does the estradiol level on the day of human chorionic gonadotrophin administration have an impact on pregnancy rates in patients treated with rec-FSH/GnRH antagonist? Hum Reprod. 2009;24:2902-9.

22. $\mathrm{Ng} \mathrm{EH}$. What is the threshold value for serum estradiol levels associated with adverse IVF outcomes? Fertil Steril. 2000;73:1071-2.

\section{Figures}

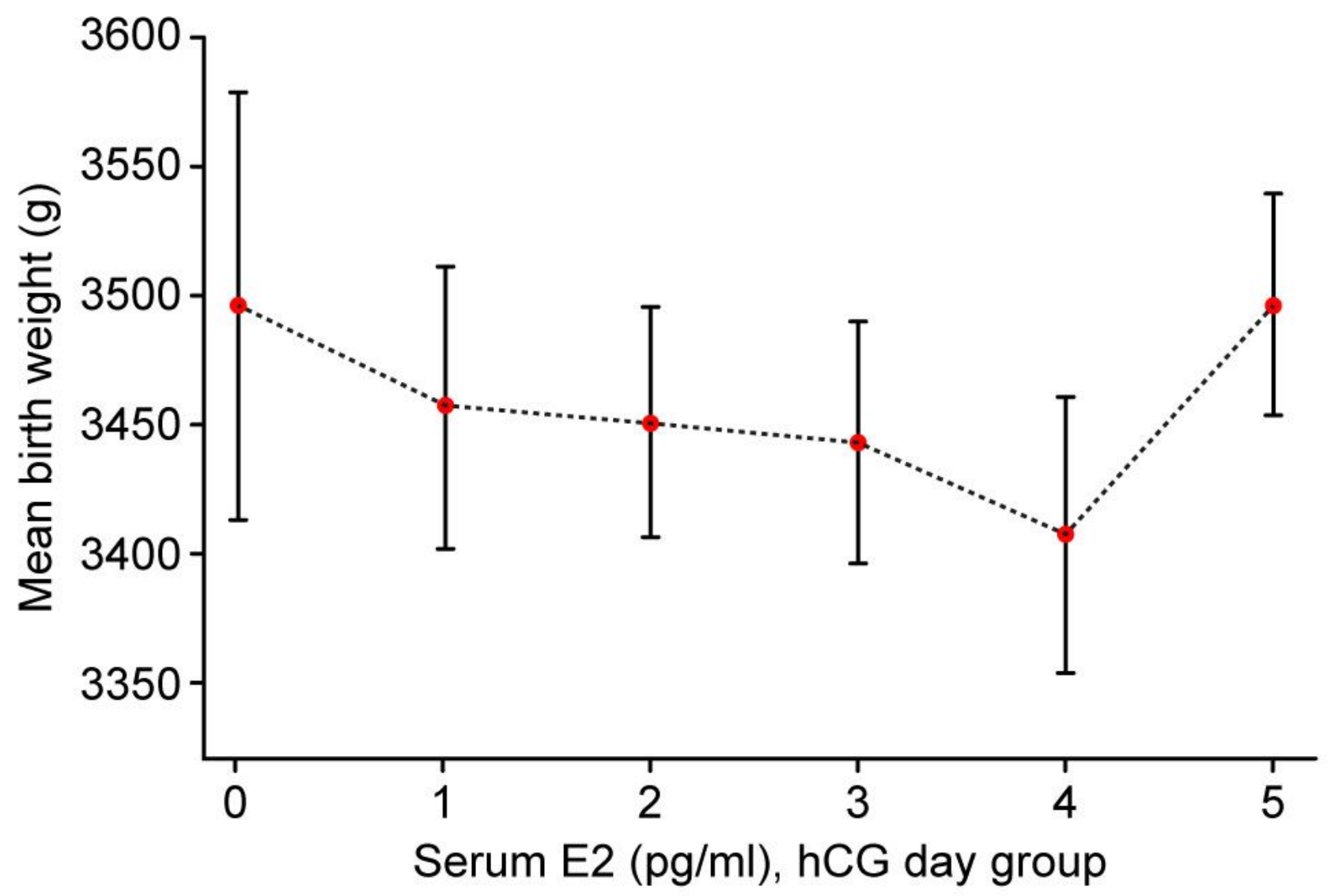

Figure 1

Page $10 / 12$ 
Relationship between serum E2 levels and neonatal birthweight.

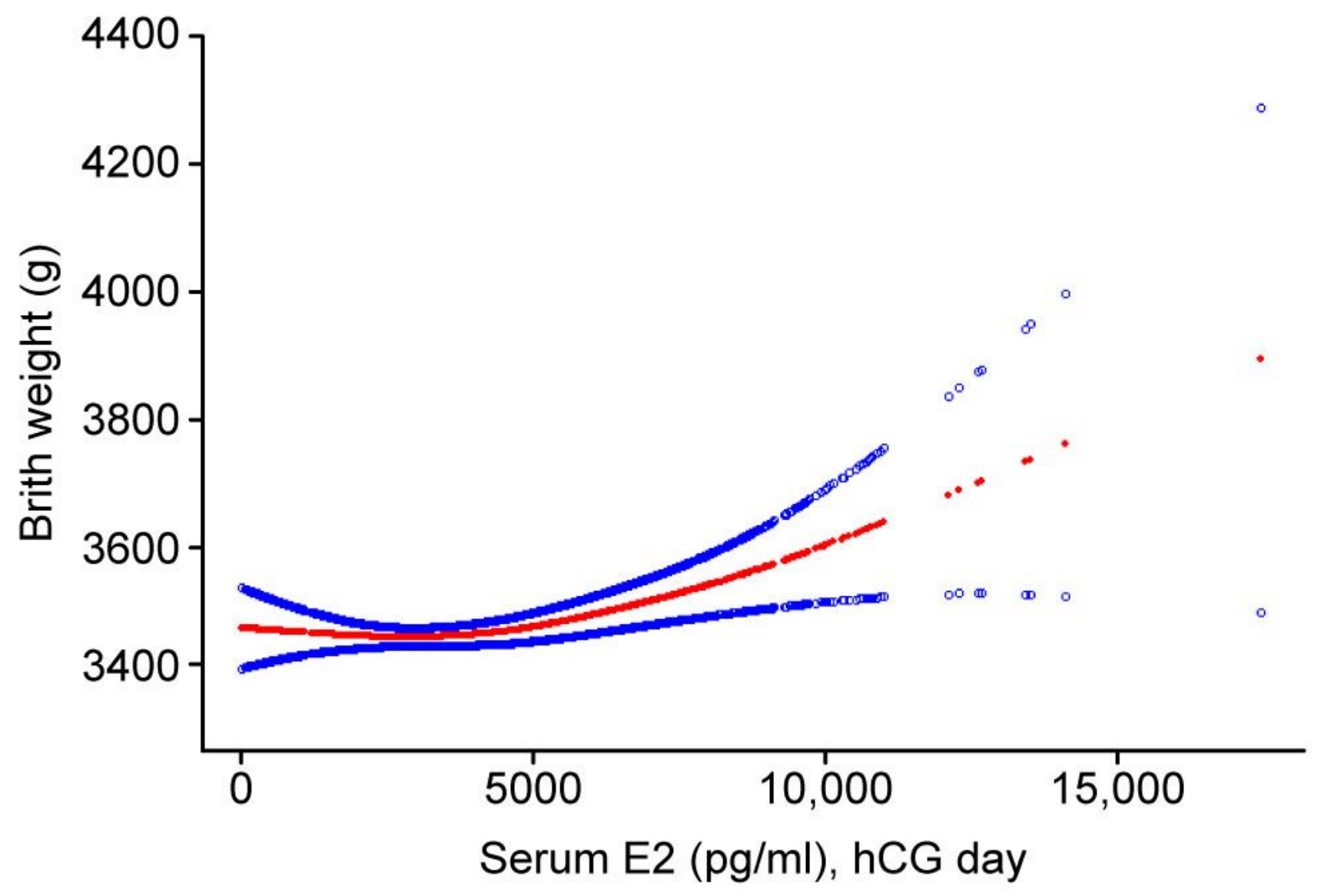

Figure 2

The relationship between serum E2 levels on the trigger day and neonatal birthweight. A nonlinear relationship between serum E2 levels and neonatal birthweight was observed after adjusting for maternal age, maternal BMI, number of embryos transferred, neonatal sex. 


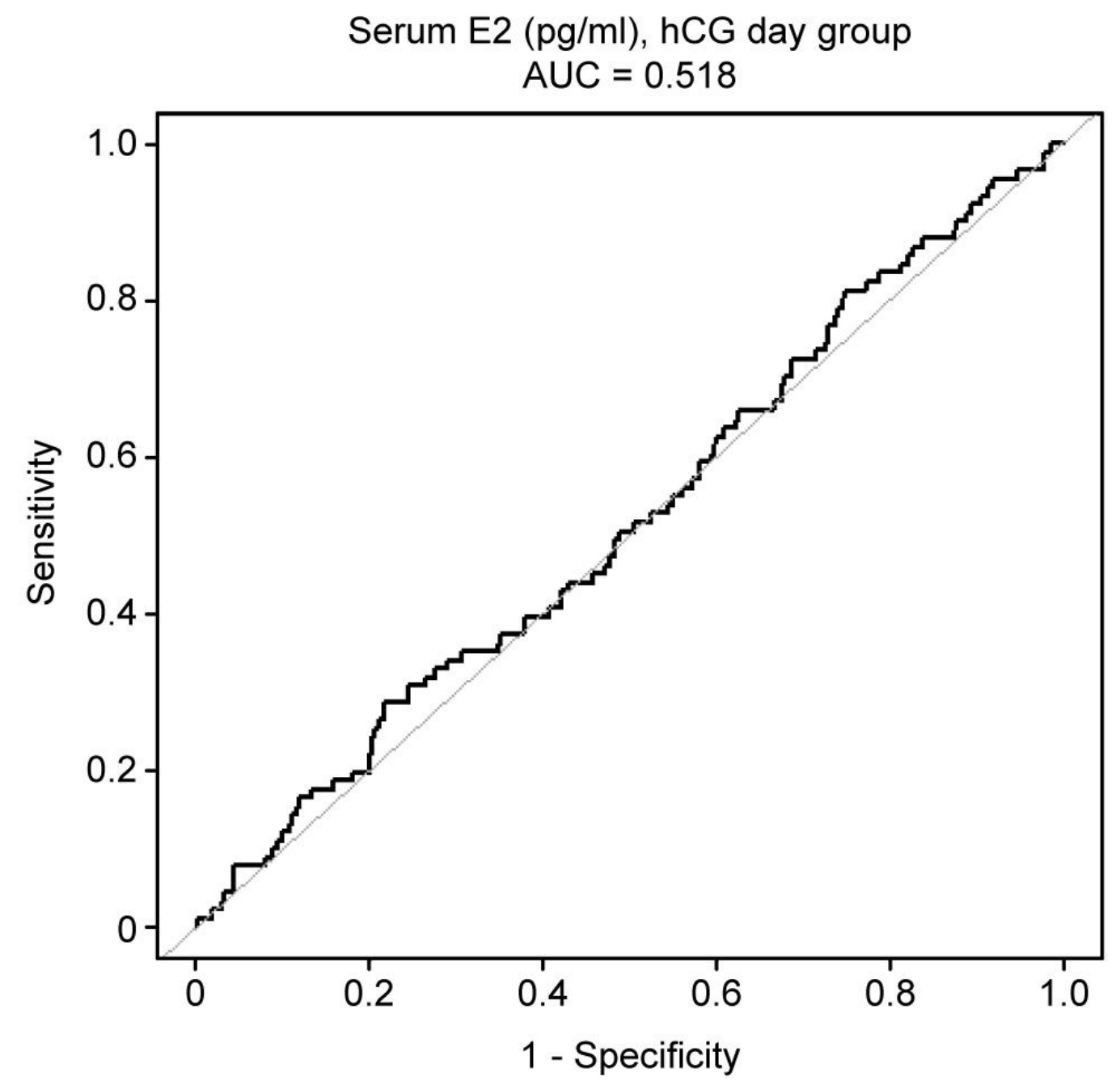

Figure 3

Receiver operating characteristic (ROC) curve evaluation of the ability of peak E2 measurements to predict low birth weight. The best threshold is $2062 \mathrm{pg} / \mathrm{ml}$ with a sensitivity of $28.6 \%$, a specificity of $78.1 \%$, and an area under the curve of 0.518 . 